\title{
Tien Shan Astronomical Observatory
}

\author{
KENESKEN KURATOV \\ Tien Shan Astronomical Observatory \\ Fesenkov Astrophysical Institute, Almaty, Kazakhstan
}

The instruments, astroclimate and research perspectives of the Tien Shan Astronomical Observatory are reviewed.

Tien Shan Astronomical Observatory (TSAO) was formed in 1994 by fusing the Solar Observatory of Fesenkov Astrophysical Institute (Republic of Kazakhstan) with the former Tien Shan Mountain Expedition of Shternberg Astronomical Institute (SAI), Moscow State University (Russia). At present work is being carried out to organize a joint Kazakh-Russian Astronomical Observatory on the basis of the structures and astronomical instruments of TSAO. We are interested in the participation of astronomical organizations from other countries, and any such proposals will be considered.

Tien Shan Astronomical Observatory is located $20 \mathrm{~km}$ from the southern boundary of Almaty city at altitudes of 2750 to $2780 \mathrm{~m}$ above sea level. Thus the Observatory combines the advantages of considerable altitude and the proximity of a large city.

The main astronomical telescopes available at the Observatory are:

Two 1-m Ritchey-Chrétien/Coudé telescopes (Zeiss-1000)

Two 48-cm Cassegrain telescopes

The HSFA horizontal solar telescope-spectrograph (Carl Zeiss Jena), with a $60-\mathrm{cm}$ mirror.

Besides the instruments mentioned, the Observatory is equipped with a $20-\mathrm{cm}$ Coudé refractor (Opton), an ACU-5 horizontal solar telescope with an ASP20 spectrograph, a large Nikolsky coronograph (diameter of main objective 53 $\mathrm{cm}$ ), and a super illumination Schmidt astrograph (Schmidt plate diameter $40 \mathrm{~cm}$, illumination 1:2). An 80-cm Carl Zeiss automated reflector is being mounted at present. 